\title{
Effect of the Configuration of Poly(methacrylic acid) on the Complex Formation with Poly-L-lysine
}

\author{
Katsuro Shinoda, Kazuhiko SAKaI, Toshio Hayashi, \\ and Akio NakaJima* \\ Department of Polymer Chemistry, Kyoto University, \\ Sakyo-ku, Kyoto 606, Japan. \\ (Received October 28, 1975)
}

\begin{abstract}
The effect of the configuration of poly(methacrylic acid) (PMA) on the complex formation with poly-L-lysine (PLL) in aqueous media has been studied by circular dichroism (CD) spectroscopy. Four kinds of polymethacrylic acids, i.e., isotactic (iso), syndiotactic (synd), atactic (at), and conventional (conv) PMA's, were used. The results indicate that the coexisting iso-PMA always induces $\alpha$-helix formation for PLL, while the other PMA's do not. Particularly, in the large excess of PMA fraction, PMA's other than iso-PMA disturb the helix formation of PLL even at $\mathrm{pH} 11$, where PLL by itself takes the $\alpha$-helical conformation. The amount by which PMA disturbs the helix formation of PLL is in the order of increasing strength: iso $\ll$ synd $<$ conv $\leq$ at. This order is parallel to the "degree of atacticity" of PMA. These results, together with the induced CD spectrum data measured by using acridine orange as a chromophore, suggest that the structures of the complexes may be classified into two kinds. One is the structure in which the PMA molecule binds to the right-handed $\alpha$-helix of PLL in such a manner as to form a super helix around the core; the other is the structure in which both component polymers form random aggregates. The ratio of these structures would depend on the configuration and the mixing ratio of PMA and PLL, as well as on the $\mathrm{pH}$ of the surrounding aqueous solutions.

KEY WORDS Polyelectrolyte Complex / Poly-L-lysine / Poly(methacrylic acid) / Configurations / Tacticity / Conformations / Circular Dichroism / Induced Circular Dichroism /
\end{abstract}

In a previous paper, ${ }^{1}$ we reported that poly(acrylic acid) interacted with poly-L-lysine (PLL) stoichiometrically in aqueous solution. However, several authors reported some polyacid-PLL systems which do not obey the stoichiometry. ${ }^{2-4}$ Rifkind, et al., ${ }^{2}$ studied the interactions of various ribonucleotides with various basic polypeptides from the measurements of optical rotatory dispersion and concluded that these reactions depend on the geometry and the structure of the involved particular nucleotides and amino acids. Gelman, et al., ${ }^{3}$ also reported the effect of the location and the type of ionizable groups on the interactions of basic polypeptides with acidic mucopolysaccharides. A similar result was reported by Roychoudhyry, et al., ${ }^{4}$ for the PLL-phosvitin system.

* To whom correspondence should be addressed.
In this paper, the effect of the configuration of poly(methacrylic acid) (PMA) on the interactions with PLL is investigated by using circular dichroism spectroscopy. To date, as far as we know, only one study has been reported about PLL-PMA interactions. Katchalsky ${ }^{5}$ pointed out from turbidimetric measurements that PLL interacts with PMA stoichiometrically. However, we will show that there exist some configurational specificities on the PLL-PMA interactions.

\section{EXPERIMENTAL}

\section{Materials}

The results of the characterization of the samples are shown in Table I. The synthetic methods were as follows. PLL was the same sample as previously described. ${ }^{6}$ Conventional PMA (conv-PMA) was obtained by radiation 
Complex of Poly-L-lysine with Poly(methacrylic acid)

Table I. Characterization of polymers

\begin{tabular}{|c|c|c|c|c|c|c|}
\hline \multirow{2}{*}{ Sample } & \multirow{2}{*}{$\overline{\mathrm{DP}}$} & \multicolumn{3}{|c|}{ Tacticity } & \multirow{2}{*}{$\sigma$} & \multirow{2}{*}{$\mathrm{p} K_{\mathrm{a}}$} \\
\hline & & $1 \%$ & $\mathbf{H} \%$ & $\mathrm{~S} \%$ & & \\
\hline conv-PMA & 760 & 5 & 32 & 63 & 0.24 & 6.25 \\
\hline synd-PMA & 80 & 3 & 13 & 84 & 0.17 & 6.07 \\
\hline at-PMA & 460 & 12 & 48 & 40 & 0.35 & 6.45 \\
\hline iso-PMA & 1100 & 100 & & & & 6.55 \\
\hline PLL & 710 & & & & & 9.40 \\
\hline
\end{tabular}

polymerization at $20^{\circ} \mathrm{C}$ in methanol with $\mathrm{Co}^{60}$ $\gamma$-rays. Syndiotactic PMA (synd-PMA) was prepared by $\gamma$-ray polymerization of methacrylic acid at $-78^{\circ} \mathrm{C}$ in methanol. ${ }^{7}$ Atactic PMA (at-PMA) was prepared by hydrolysis of the polymethacrylic anhydride obtained by $\gamma$-ray polymerization of methacrylic anhydride at $30^{\circ} \mathrm{C}$ in benzene. ${ }^{8}$ Isotactic PMA (iso-PMA) was prepared by hydrolysis in sulfuric acid of the isotactic poly(methyl methacrylate) obtained by the polymerization of methyl methacrylate at $0^{\circ} \mathrm{C}$ in toluene with phenyl magnesium bromide initiator. ${ }^{9,10}$ The degree of hydrolysis of both at- and iso-PMA was almost $100 \%$, as estimated from elementary analyses and conductometric titrations. The molecular weights of the PMA samples were estimated from the limiting viscosity numbers $[\eta]$ in $0.002 N \mathrm{HCl}$ aqueous solution for synd-, at-, and conv-PMA, and in chloroform solution for remethylated iso-PMA. The triad tacticity of PMA was determined by nuclear magnetic resonance after the methylation with diazomethane. The probability of an isotactic placement monomer units during the propagation, ${ }^{11} \sigma$, is shown in the third column of Table I. The proportion I: $\mathrm{H}: \mathrm{S}$ of perfectly atactic polymer will be $1: 2: 1$, at $\sigma=0.5$, which corresponds to random propagation. Acridine orange (AO) was the same sample as previously described. ${ }^{1}$

\section{Preparation of Mixtures}

Separate dilute aqueous solutions were prepared with PLL and PMA. The PLL solution was adjusted at $\mathrm{pH} 12$ by adding $1.0-N$ $\mathrm{NaOH}$. Mixtures were prepared by slow dropwise addition of an aqueous solution of PMA at $\mathrm{pH} c a .7$ to the PLL solution at $\mathrm{pH} \mathrm{12}$; then the $\mathrm{pH}$ of the mixture was brought to the desired values by adding $\mathrm{HCl}$, and neutralized $0.01-N$ AO aqueous solution was added if necessary. The relative proportions of the two polymer components are indicated in the residue-mole ratio. Some mixtures showed slight turbidity especially in the vicinity of the stoichiometric composition at the given $\mathrm{pH}$. However, the CD spectrum seems to be affected by the turbidity hardly at all (see the following paper $^{12}$ ). It is well known that significant precipitation occurs in aqueous solution when isotactic PMA is allowed to stand at low $\mathrm{pH}$ (below 5.5). However, the addition of PLL resulted in a stable solution even at $\mathrm{pH} 2.5$. This result may show that the interaction between PLL and PMA occurs even where one of the components is only slightly ionized.

\section{Circular Dichroism}

The circular dichroism (CD) spectra were measured at $25 \pm 0.5^{\circ} \mathrm{C}$ using a JASCO J-20 CD/ORD Spectropolarimeter equipped with a quartz cell of path length $1 \mathrm{~mm}$. The PLL concentration in the mixtures was from 0.002 to $0.003 \mathrm{M}$. The concentrations were determined by conductometric titration and/or gravimetry. The residue ellipticity $[\theta]$, in degree $\cdot \mathrm{cm}^{2} \cdot \mathrm{dmol}^{-1}$, for the mixtures was calculated based on the mole concentration of the PLL, except in the systems including AO where the mole concentration of AO was used.

\section{pH Measurements}

The $\mathrm{pH}$ values of the solutions were measured with a Hitachi-Horiba pH-Meter Model F-7 ss $_{\text {s }}$ equipped with a combination $\mathrm{pH}$ electrode 6028-10T, the sensitivity of which was $0.005 \mathrm{pH}$. $\mathrm{pH}$ titrations were performed in the presence of $0.02 \mathrm{M} \mathrm{NaCl}$ under nitrogen atmosphere in order to determine the state of charge of PLL and PMA under the same conditions as in the CD measurements.

\section{RESULTS AND DISCUSSION}

\section{Circular Dichroism Spectra of PLL-PMA Mix- tures}

PLL was reacted with various PMAs having different tacticities, and the $\mathrm{CD}$ spectra were measured at different $\mathrm{pH}$ values in the region of wavelengths from 200 to $250 \mathrm{~nm}$. Typical 


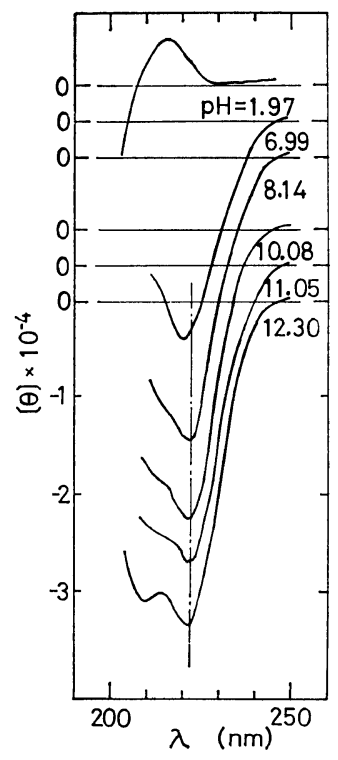

Figure 1. CD spectra of $25: 75$ mole ratio PLLisotactic PMA mixtures at $25^{\circ} \mathrm{C}$ and various $\mathrm{pH}$ values. Broken lines in Figures 1 to 5 indicate the position of $222 \mathrm{~nm}$.

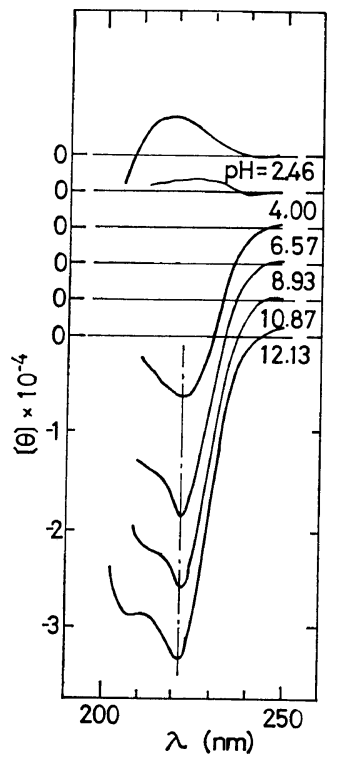

Figure 2. CD spectra of $25: 75$ mole ratio PLLsyndiotactic PMA mixtures at $25^{\circ} \mathrm{C}$ and various $\mathrm{pH}$ values.

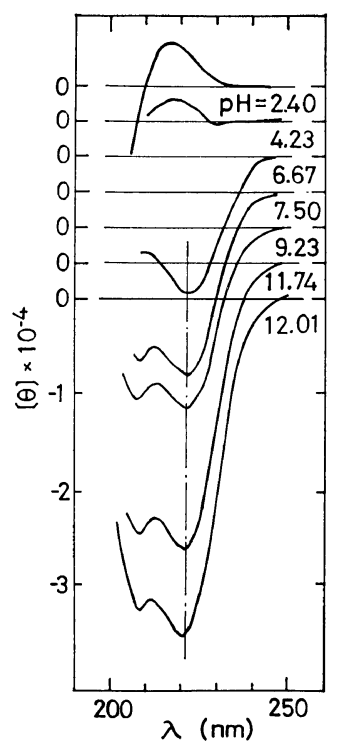

Figure 3. CD spectra of $25: 75$ mole ratio PLLconventional PMA mixtures at $25^{\circ} \mathrm{C}$ and various $\mathrm{pH}$ values.

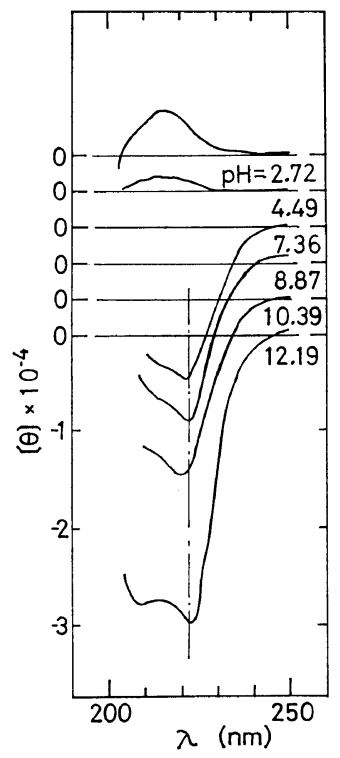

Figure 4. CD spectra of $25: 75$ mole ratio PLLatactic PMA mixtures at $25^{\circ} \mathrm{C}$ and various $\mathrm{pH}$ values. 


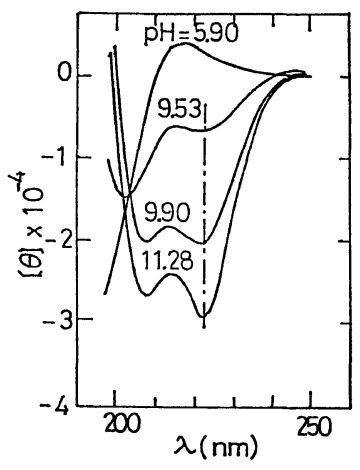

Figure 5. CD spectra of PLL in aqueous solution at $25^{\circ} \mathrm{C}$ and various $\mathrm{pH}$ values.

CD curves for various PMA-PLL mixtures containing $25 \mathrm{~mol} \%$ PLL are shown in Figures 1 to 4 . These spectra would reflect the conformation of PLL in the complex, because PMA has no strong absorption band in this region. For comparison with these curves, the CD spectra of PLL in aqueous solution at typical pH's are also shown in Figure 5. As clarified by Holzwarth and Doty, ${ }^{13}$ the $\mathrm{CD}$ spectrum of the $\alpha$-helix conformation is characterized by a negative $222 \mathrm{~nm}$ band assigned to the $\mathrm{n}-\pi^{*}$ transition, and a positive $190 \mathrm{~nm}$ band and a negative $208 \mathrm{~nm}$ band, both assigned to the $\pi-\pi^{*}$ transition. A positive band at around $218 \mathrm{~nm}$ is attributed to the "charged-coil" form of PLL; however, the structure is not well known. Our data given in Figure 5 are consistent with these results. The $\beta$-structure of PLL is characterized by a negative $217 \mathrm{~nm}$ band $([\theta]=-19,300)$ and a positive $195 \mathrm{~nm}$ band $([\theta]=+28,000) \cdot{ }^{14}$

As shown in Figures 1 to 4, the CD spectra of PMA-PLL mixtures could be classified into three categories. The first category contains the curves for a typical $\alpha$-helix or charged-coil, as shown in conv-PMA-PLL system over the entire $\mathrm{pH}$ region (Figure 3), and in other systems at the low and high $\mathrm{pH}$ regions. In this case, the PLL in the complex would exist in the normal $\alpha$-helical or charged-coil conformation. The second one shows a pattern in which the $222 \mathrm{~nm}$ band characteristic of the $\alpha$-helical conformation assigned to the $\mathrm{n}-\pi^{*}$ transition is obviously maintained, but the
$208 \mathrm{~nm}$ band assigned to the $\pi-\pi^{*}$ transition is a bit uncertain. These curves are observed in Figure $1(\mathrm{pH}=8.14,10.08$, and 11.05), Figure $2(\mathrm{pH}=6.57,8.93$, and 10.87), and Figure $4(\mathrm{pH}=7.36$ and 8.87$)$. The $\pi-\pi^{*}$ transition originates mainly from the main chain structure, while the $n-\pi^{*}$ transition originates from the relative conformation of the side chain residue to the peptide plane. From these points of view, these curves may suggest that the PLL in the complex would take a slightly distorted $\alpha$-helical conformation. This may be caused by the location of the ionized carboxyl group on PMA relative to the $\varepsilon$-amino on PLL. The third category is found in Figure $1(\mathrm{pH}=6.99)$ and Figure $4(\mathrm{pH}=10.39)$, in which a negative band appears at $220 \mathrm{~nm}$. This pattern may suggest that the PLL would take a $\beta$-like extended conformation or would be a mixture of $\alpha$-helix and $\beta$-structure. However, as will be indicated later, the numerical value of the residue ellipticity at $222 \mathrm{~nm}$ changes smoothly with $\mathrm{pH}$ values, in spite of the change in $C D$ patterns. This may indicate that the third structure does not largely differ from the $\alpha$-helix.

The CD spectra of other mixtures having different PMA-PLL mole radios at various pH's gave similar results. It should be noted that the configurational specificity of PMA does not appear in the conformation of PLL in the complex. In a previous paper, ${ }^{1}$ we reported that most of the CD curves of poly(acrylic acid) (PAA)-PLL mixtures showed spectra typical of the $\alpha$-helix or charged coil. The difference in the CD curves observed between PMA-PLL and PAA-PLL seems to be dependent on the diversity in the relative location of the carboxyl group to the $\varepsilon$-amino group, due to the difference in chain flexibility.

pH Dependence of Residue Ellipticity at $222 \mathrm{~nm}$ for Various PMA-PLL Systems

In Figures 6 to 9, the residue ellipticity at $222 \mathrm{~nm},-[\theta]_{222}$, of the mixtures containing different ratios of various PMA and PLL is plotted against $\mathrm{pH}$, here data not shown in previous figures are also included. As indicated in these figures, PLL shows a sharp transition at about $\mathrm{pH} 9.5$, but the situation is remarkably 


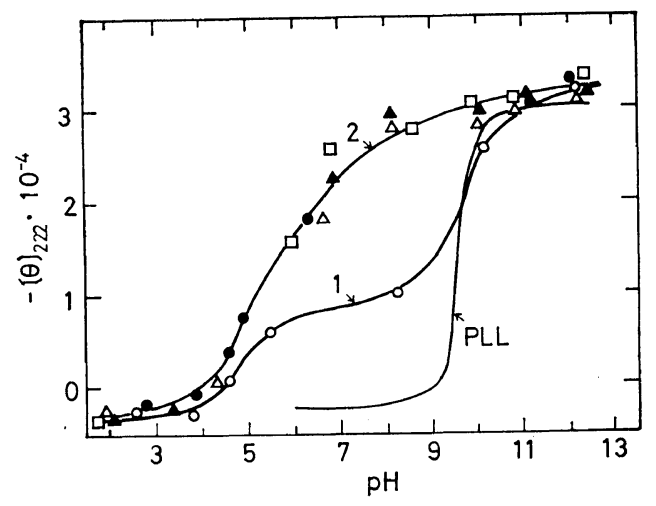

Figure 6. $-[\theta]_{222}$ plotted against $\mathrm{pH}$ for PLLisotactic PMA mixtures and for PLL at $25^{\circ} \mathrm{C}$. The mole ratios of PLL to iso-PMA are: (1) $67: 33$; (2) $50: 50, \triangle 33: 67, \triangle 25: 75$, $\square 11: 89$.

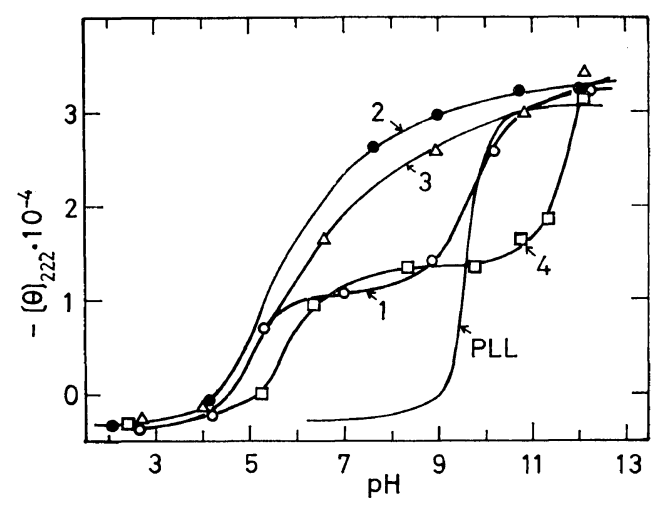

Figure 7. $-[\theta]_{222}$ plotted against $\mathrm{pH}$ for PLLsyndiotactic PMA mixtures and for PLL at $25^{\circ} \mathrm{C}$. The mole ratios of PLL to synd-PMA are: (1) $66: 34$; (2) $33: 67$; (3) $25: 75$; (4) $10: 90$.

changed by the coexistence of PMA's. Moreover, the situations are greatly affected by the configuration of PMA. Iso-PMA interacts with PLL and induces the formation of a helix of PLL in proportion to the mole fraction and the degree of dissociation, in a manner similar to the PAA-PLL systems. This phenomenon would be interpreted as a result of the disappearance of the electrostatic repulsion due to the neutralization of the $\varepsilon$-amino groups of PLL by the ionized carboxyl groups of PMA. On the other hand, PMA-PLL systems other than the iso-PMA-PLL system have some common

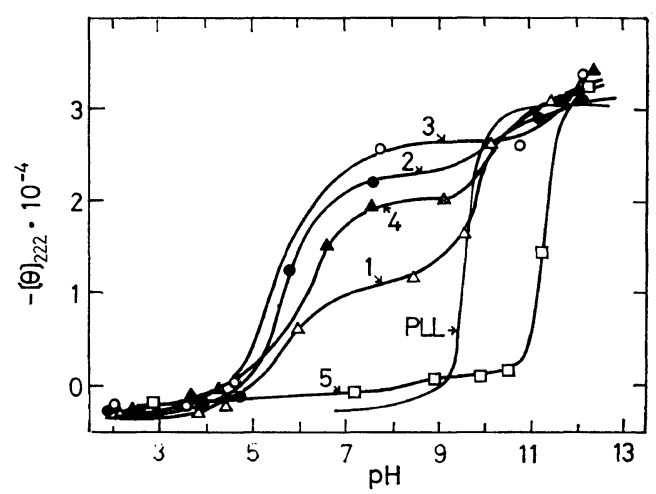

Figure 8. $-[\theta]_{222}$ plotted against $\mathrm{pH}$ for PLLconventional PMA mixtures and for PLL at $25^{\circ} \mathrm{C}$. The mole ratios of PLL to conv-PMA are: (1) $69: 31$; (2) $53: 47$; (3) $33: 67$; (4) $25: 75$; (5) $13: 87$.

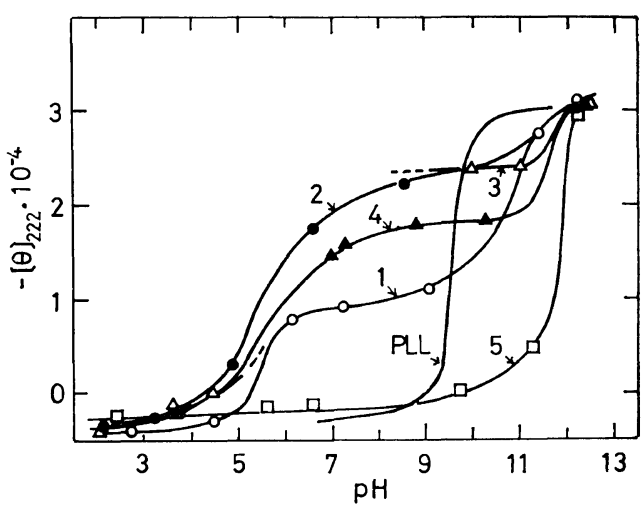

Figure 9. $-[\theta]_{222}$ plotted against $\mathrm{pH}$ for PLLatactic PMA mixtures and for PLL at $25^{\circ} \mathrm{C}$. The mole ratios of PLL to at-PMA are: (1) $67: 33$; (2) $50: 50$; (3) $34: 66$; (4) $25: 75$; (5) $10: 90$.

aspects, that is, the value of $-[\theta]_{222}$ shows a progressive change with PMA-PLL mole ratio. In the region of relatively low mole fraction of PMA, the helical conformation of PLL is induced by PMA in a manner similar to the iso-PMA-PLL system. In the region of high mole fraction of PMA, however, the helix formation of PLL is disturbed by PMA even at $\mathrm{pH} 10.5$, where PLL by itself can form an $\alpha$-helix. This behavior can be seen more clearly in the plot of $-[\theta]_{222}$ against the mole fraction of PMA at $\mathrm{pH} 7.5$, where both components are almost fully ionized. (Figure 10) 


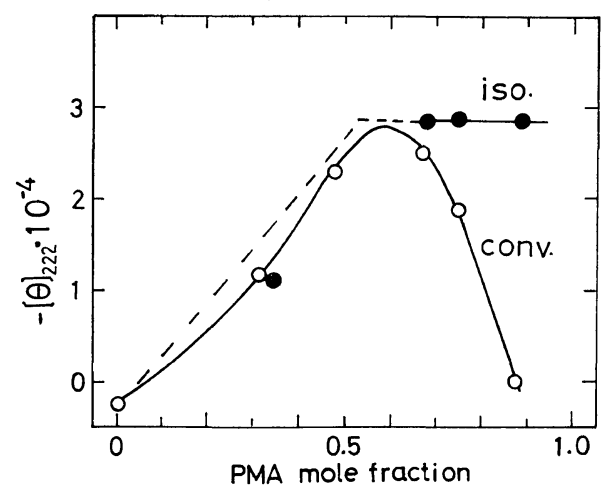

Figure 10. $-[\theta]_{222}$ plotted against PMA mole fraction at $\mathrm{pH} 7.5$ for PLL-iso-PMA and for PLL-conv-PMA mixtures.

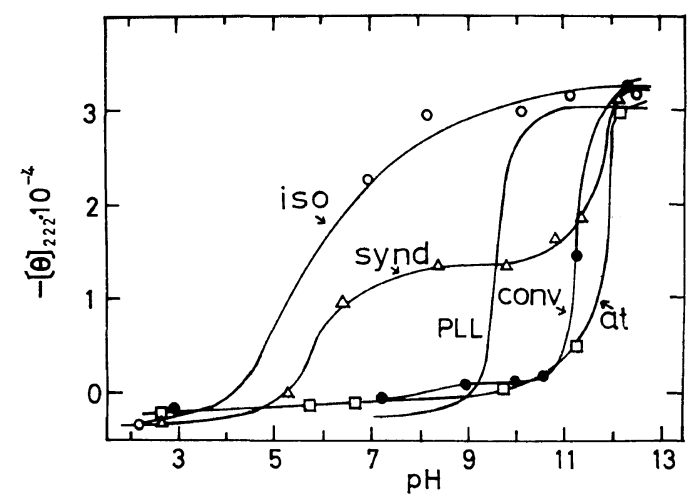

Figure 11. $-[\theta]_{222}$ plotted against $\mathrm{pH}$ for various PMA (ca. 90 mol-\%)-PLL (ca. 10 mol-\%) mixtures.

Obviously, conv-PMA (also synd- and at-PMA) has two competitive effects: i.e., the effects which induce and which disturb the helix formation of PLL in the mixtures.

In order to examine the effect of the nature of PMA on the conformation-directing interaction, $-[\theta]_{222}$ is plotted against $\mathrm{pH}$ for various PMA (90 mol- $\%)$-PLL (10 mol- $\%)$ mixtures in Figure 11. As is obvious from the figure, the strength of the disturbing effect on the helix formation of PLL is in the order: iso $\ll$ synd $<$ conv $\leq$ at. This order, as is expected from the potentiometric titration curves (Figure 12), may be related not to the nature of the dissociation a of the PMA samples but to the "degree of atacticity" of the samples (see the values of $\sigma$

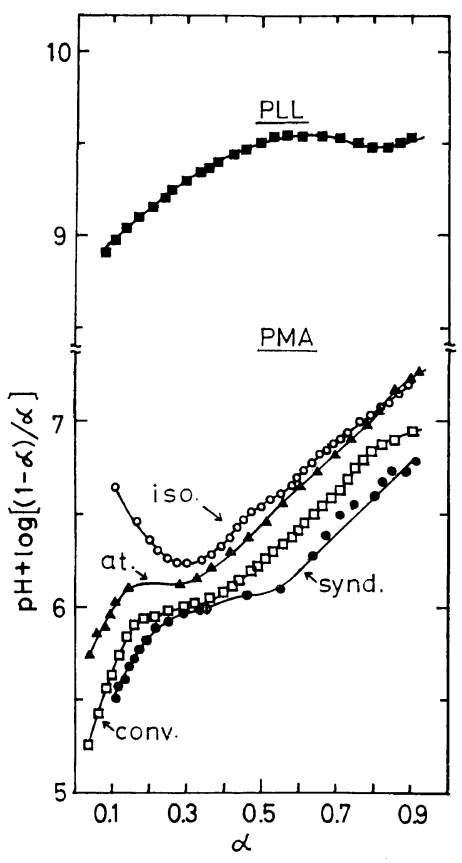

Figure 12. Potentiometric titration curves of PLL and various $\mathrm{PMA}$ 's at $25^{\circ} \mathrm{C}$ : Polymer concn, $0.0025 N ; \mathrm{NaCl}$ concn, $0.02 N$.

in Table I). As shown in Table I, the molecular weights of these four PMA samples are not equal. However, in a previous paper, ${ }^{1}$ we reported that the effect of molecular weight of PAA on the PAA-PLL system was negligibly small over the $\overline{\text { DP }}$ range from 80 to 3,100 . Thus, we conclude that these effects would be attributed mainly to the configurations of PMA.

With respect to the disturbing effect of an oppositely charged polyelectrolyte on the helix formation of a polypeptide containing ionizable groups, some results have been reported., ${ }^{3,15}$ However, the mechanism has not been clarified. In our case, the following interpretation may be possible by taking into consideration the effect of molecular chain configurations. When a polyanion reacts with a polycation, the charged groups would not always be neutralized by the oppositely charged groups. An unsuited mutual location of oppositely changed groups on the polycation and polyanion chains, or geometrical hindrance, may let some charged groups reamain in a free (charged) state. The repulsion forces among these free charged groups of the same 
sign will disturb the formation of a helical structure. Our results suggest that the number of such charged groups increases with increasing the "degree of atacticity".

The fact that the helix formation of PLL is induced almost proportionally to the PMA mole fraction below 0.5 PMA mol fraction (see Figure 10 conv-PMA-PLL) may suggest that the number of the free charged groups is not so large. However, the chances for free charged groups on a "free" PMA chain to bind with free $\varepsilon$-amino groups of PLL in the complex will increase with increasing PMA mole fraction. The newly bound PMA would have more free carboxyl groups than the already bound PMA. Such a large number of PMA rich in free carboxyl groups existing in the complex may cause a strong disturbing effect. This may be one of the reasons for the large disturbing effect at high PMA mole fraction.

\section{Structure of Complex}

Conformations of the PLL in the complex are deduced from CD spectra in the UV region. However, no information is given on the structure of the other component in the complex. In a previous paper, ${ }^{1}$ we suggested the structure of PAA in the PAA-PLL complex

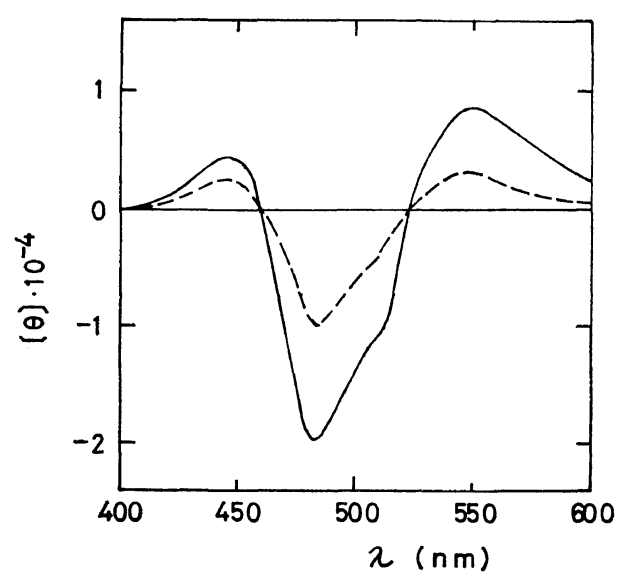

Figure 13. CD spectra of PLL-synd-PMA-AO systems at $25^{\circ} \mathrm{C}$ and $\mathrm{pH}$ 6.18. Molar ellipticity is denoted based on the molar concentration of AO. Concentrations: - $[\mathrm{PLL}]=0.0018 \mathrm{~N}$, [synd$\mathrm{PMA}]=0.01060 \mathrm{~N},[\mathrm{AO}]=0.0014 \mathrm{~N} ;-\cdots,--,[\mathrm{PLL}]=$ $0.00144 N$, [synd-PMA] $=0.01230 N,[$ AO] $=0.00140$ $N$. system by using the technique of induced $C D$. For the present system, we applied the same technique. Figure 13 shows the induced $C D$ spectra on the systems of synd-PMA-PLL-AO. PMA is known to exhibit conformational change from a compact to an extended mean conformation, in accordance with the degree of ionization. ${ }^{17}$ Koenig, et al. ${ }^{18}$ indicated that syndiotactic PMA took a helical conformation in the solid state. Nagasawa, et al. ${ }^{19}$ from the results of potentiometric titration, suggested that the syndiotactic PMA existed in a locally planer zigzag conformation, and isotactic PMA a locally helical conformation in aqueous solution. However, we have not found any characteristic spectrum for the various PMAAO systems of various mole ratios, over a wide $\mathrm{pH}$ region. This may indicate that the PMA samples we used do not exist in any ordered structure at least spectropolarimetrically under ordinary conditions. The CD curves in Figure 13 are similar to those of the poly(glutamic acid)-AO system ${ }^{20}$ and the CMC-AO system, ${ }^{21}$ and may indicate a right-handed super helical structure of the PMA. The bands at 445 and $485 \mathrm{~nm}$ may be responsible for the electronic transitions polarized parallel and prependicular, respectively, to the axis of the helix, by taking into consideration the result for the poly(glutamic acid)-AO system. With respect to the positive band at $545 \mathrm{~nm}$, its corresponding negative band may be observed as a shoulder at around $520 \mathrm{~nm}$, though it has not been assigned. Still, this band may be understand-

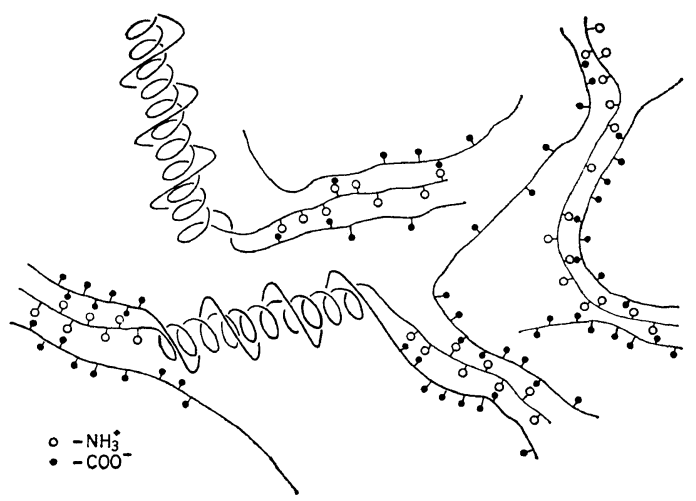

Figure 14. Schematic models of PLL-PMA complexes. 
able if we assume that monomeric AO is bound in the complex and forms a left-handed helical array. Such a structure is deduced from the fact that the monomeric AO is intercalated between the base pairs of DNA and induces a $\mathrm{CD}$ band at around $500 \mathrm{~nm}$ in the DNA-AO system. ${ }^{22,23}$

These results lead to the conclusion that the structures of the complex may be classified into two categories: one is the structure in which the PMA molecule binds to the right-handed $\alpha$-helix of PLL in such a manner as to form a super helix surrounding the core; the other is the structure in which both polymer components form random aggregates, as shown in Figure 14. In the former case, the charged groups would be neutralized almost stoichiometrically by the oppositely charged groups, while, in the latter case, many free charged groups would be remaining in the random aggregates due to the inadequate mutual location of oppositely charged groups. The ratio of these two structures would depend on the configuration and mole fraction of the PMA as well as on the $\mathrm{pH}$ of the surrounding solution.

Poly(aerylic acid) reacts with PLL stoichiometrically. ${ }^{1}$ The difference in the stoichiometry between the PMA-PLL and PAA-PLL systems may be due to the difference in chain flexibility. The methyl groups attached to the backbone chain of PMA would increase the stiffness of the chain in aqueous solution.

In conclusion, we point out that the coufiguration of the component polymers is one of the important factors needed to discuss the formation and structure of such polyelectrolyte complexes as described here.

Acknowledgment. The authors wish to thank Dr. H. Kamiyama, Mr. H. Ogawara, and Mr. T. Nakagawa, Sekisui Chemical Co., for NMR measurements and radiation polymerization.

\section{REFERENCES}

1. K. Shinoda, T. Hayashi, T. Yoshida, K. Sakai, and A. Nakajima, Polymer J., 8, 202 (1976).

2. J.M. Rifkind and G. L. Eichhorn, Biochemistry, 9, 1753 (1970).

3. R. A. Gelman and J. Blackwell, Biopolymers, 13, 139 (1974).

4. A. Roychandhyry, B. B. Biseas, and M. K. Pal, Makromol. Chem., 124, 113 (1969).

5. A. Katchalsky, Biophys. J., 4, 9 (1964).

6. A. Nakajima, K. Shinoda, T. Hayashi, and H. Sato, Polymer J., 7, 550 (1975).

7. T. Matsuda, H. Yamashita, and Y. Sakai, Kobunshi Kagaku (Chem. High Polymers), 20, 614 (1963).

8. W. L. Miller, W. S. Brey, Jr., and G. B. Butler, J. Polym. Sci., 54, 329 (1961).

9. E. M. Loebl and J. J. O'Neill, J. Polym. Sci., 45, 538 (1960).

10. J. Semen and J. B. Lando, Macromolecules, 2 , 570 (1969).

11. F. A. Bovey and G. V. D. Tiers, J. Polym. Sci., 44, 173 (1960).

12. K. Shinoda, T. Hayashi, and A. Nakajima, Polymer J., to be submitted.

13. G. Holzwarth and P. Doty, J. Amer. Chem. Soc., 87, 218 (1965).

14. B. Davidson, N. Tooney, and G. D. Fasman, Biochem. Biophys. Res. Commun., 23, 156 (1966).

15. A. B. Zezin, V. V. Lutsenko, V. B. Rogacheva, O. A. Aleksina, R. I. Kslyuzhnaya, V. A. Kavanov, and V.A. Kargin, Vysokomol. Soedin., A14(4), 772 (1972),

16. R. A. Gelman and J. Blackwell, Biopolymers, 12, 1959 (1973).

17. M. Mandel, J.C. Leyte, and M. G. Stadhouder, J. Phys. Chem., 71, 603 (1967).

18. J. L. Koenig., A. C. Angood, J. Semen, and J. B. Lando, J. Amer. Chem. Soc., 91, 7250 (1969).

19. M. Nagasawa, T. Murase, and K. Kondo, $J$. Phys. Chem., 69, 4005 (1965).

20. M. Hatano, M. Yoneyama, and Y. Sato, Biopolymers, 12, 895 (1973).

21. K. Nishida and A. Iwasaki, Kolloid-Z.Z. Polyme., 251, 136 (1973).

22. B. J. Gardner and S. F. Mason, Biopolymers, 5, 79 (1967).

23. M. Zama and S. Ichimura, ibid., 9, 53 (1970). 